Regards sur l'économie allemande

Bulletin économique du CIRAC

$68 \mid 2004$

Varia

\title{
Pas d'impôt apprentissage, mais un Pacte pour la formation
}

\section{Brigitte Lestrade}

\section{(2) OpenEdition}

Journals

Édition électronique

URL : http://journals.openedition.org/rea/3622

DOI : $10.4000 /$ rea.3622

ISBN : 978-2-8218-0832-4

ISSN : 1965-0787

Éditeur

CIRAC

Édition imprimée

Date de publication : 1 octobre 2004

Pagination : 17-20

ISSN : 1156-8992

Référence électronique

Brigitte Lestrade, «Pas d'impôt apprentissage, mais un Pacte pour la formation », Regards sur l'économie allemande [En ligne], 68 l octobre 2004, mis en ligne le 29 avril 2009, consulté le 19 avril 2019. URL : http://journals.openedition.org/rea/3622 ; DOI : 10.4000/rea.3622 


\title{
Pas d'impôt apprentissage, mais un Pacte pour la formation
}

\author{
Brigitte Lestrade
}

Depuis les débuts de l'industrialisation en Allemagne, la formation professionnelle est considérée comme relevant de la responsabilité des entreprises. Responsabilité lourde, car la très grande majorité des jeunes choisit une formation duale. Ce système, entièrement financé par les entreprises qui acceptent de former des jeunes, intègre entre 550000 et 700000 nouveaux apprentis par an. Or la formation duale, qui a fonctionné à la satisfaction générale pendant si longtemps, est en crise. Depuis 2000, l'offre de places en apprentissage diminue, le nombre d'entreprises qui acceptent de former des jeunes se réduit fortement. Le gouvernement fédéral, soucieux de l'accueil désastreux fait à l'Agenda 2010 et désireux de récupérer une image "sociale ", avait proposé fin 2003 de créer un impôt qui frapperait les entreprises n'assumant pas leurs responsabilités en matière de formation. Retiré, le projet aura débouché sur un Pacte...

Le gouvernement fédéral affiche ses ambitions. II estime que le ratio offre/demande de places d'apprentissage devrait se situer nettement au-dessus de $100 \%$ (le projet de loi évoque 115\%) pour permettre à tous les jeunes qui le souhaitent de trouver la formation duale adéquate. Ce ratio très favorable qui, en pratique, n'a été atteint que pendant les trois ans qui ont suivi la chute du Mur de Berlin, s'est fortement dégradé ces dernières années. Le nombre de places d'apprentissage proposées par les entreprises est passé de 625442 en 2000 à 546660 en 2003 (IAB, 2004). Tout aussi significative est l'augmentation parallèle de l'offre de formation dans des centres agréés hors entreprises accueillant majoritairement des jeunes en difficulté, qui passe de 57314 à 61453 , soit un accroissement de plus de $7 \%$ en trois ans. Le ratio offre/demande tombe ainsi de $100,3 \%$ à $96,6 \%$ (91,2\% dans les nouveaux Länder), le plus faible niveau depuis vingt ans. Cette dégradation signifie que 30000 jeunes n'ont pas réussi à intégrer une formation duale en 2003. Comment expliquer ce retrait de l'engagement des entreprises ?

De plus en plus d'entreprises allemandes estiment que l'apprentissage leur coûte trop cher. La rémunération des apprentis d'abord, qui atteint des niveaux tels qu'elle constitue un vrai salaire leur permettant d'assurer leur subsistance. Les revenus d'un apprenti sont assortis des mêmes charges que ceux d'un salarié et bénéficient des mêmes prestations sociales volontaires. L'apprenti a en effet un statut de salarié, sa rémunération atteignant au moins $50 \%$ du salaire conventionnel en vigueur pour un débutant dans sa branche. S'y ajoutent les coûts occasionnés par les formateurs, en augmentation eux aussi en raison des contenus de formation toujours plus exigeants, ainsi que, le cas échéant, les coûts d'installation d'ateliers réservés à la formation des apprentis.

En face de cet accroissement des coûts, les entreprises constatent que les bénéfices qu'elles en retirent sont en diminution. Les jeunes ne passent en effet qu'une partie de la semaine en entreprise, deux à trois journées sur cinq, le reste étant consacré à la formation théorique à l'école professionnelle. Si le produit du travail des apprentis paraît moins intéressant aux entreprises aujourd'hui, c'est notamment dû au hiatus croissant entre les exigences toujours plus pointues des formations professionnelles actuelles et le niveau scolaire des

Recul de l'offre de places d'apprentissage

Des coûts de plus en plus élevés..

...pour un retour décroissant 
L'impôt apprentissage : une mesure pour amadouer les électeurs sociaux-démocrates...

... mais qui n'est pas nouvelle

Un monstre bureaucratique...

...sous le feu des critiques nouveaux entrants dont l'étude Pisa a montré les déficits (voir REA 66/04). Les activités d'une entreprise offrent de moins en moins de travaux simples susceptibles d'être effectués par un apprenti. A ce problème s'ajoute la situation conjoncturelle actuelle. Une entreprise sera moins tentée de former des apprentis si elle pense ne pas pouvoir les embaucher à la fin de leur formation, sachant par ailleurs que les candidats à une embauche éventuelle ne manquent pas dans un marché de l'emploi déprimé comme c'est le cas actuellement.

L'Etat s'étant de plus en plus impliqué dans la formation duale, notamment par le programme JUMP destiné à combattre le chômage des jeunes (Lestrade, 2000), il n'est pas étonnant qu'il se soit saisi de la question de la pénurie des places d'apprentissage au moment où l'Agenda 2010 tentait de réformer en profondeur un marché de l'emploi réputé trop rigide. Les mesures prévues dans le cadre de la réforme, en particulier la fusion entre l'assistance chômage et l'aide sociale au niveau plus bas de cette dernière, allaient à ce point à l'encontre des convictions des électeurs sociaux-démocrates de base, que le nouveau président du SPD, Franz Müntefering, estimait nécessaire d'introduire une mesure reflétant l'esprit social-démocrate. II proposa la création d'un impôt devant frapper toutes les entreprises qui n'offraient pas assez de places d'apprentissage. Les fonds ainsi recueillis seraient destinés à financer celles qui forment au-delà d'un certain seuil à définir.

L'idée d'un impôt pour financer la formation professionnelle revient régulièrement dans le débat politique dès que se profile un ratio demande/offre défavorable. En 1976, les sociaux-démocrates alors au gouvernement avaient fait voter par le Bundestag la Loi sur la promotion des places d'apprentissage (Ausbildungsplatzförderungsgesetz, AplfG) créant une taxe spécifique ne requérant pas l'approbation du Bundesrat, le SPD n'y ayant pas la majorité, à l'époque comme aujourd'hui. A l'issue d'une plainte déposée par le gouvernement bavarois, cette loi a été déclarée contraire à la Constitution par le Tribunal constitutionnel fédéral. Une nouvelle tentative en 1996 n'eut pas davantage de succès.

Dans son projet de loi (BT-Drucksache 15/2820), la coalition fédérale justifie son initiative par le recul constant du nombre d'entreprises formant des apprentis (23\% seulement du total en 2003 ), la menace d'une pénurie de personnel qualifié, aggravée par une démographie défavorable, ainsi que par le chômage des jeunes qui touche surtout ceux sans formation. C'est pourquoi le gouvernement souhaite que tous les jeunes à la recherche d'une formation puissent accéder à l'apprentissage. La nouvelle réglementation s'applique si, au 30 septembre de l'année considérée, le nombre total de nouvelles places d'apprentissage n'est pas supérieur d'au moins $15 \%$ à celui des jeunes qui en cherchent une, et si aucune amélioration de la situation n'est en vue. Dans ce cas, toutes les entreprises de 10 salariés et plus dont le nombre d'apprentis est inférieur à $7 \%$ du total des salariés devront alimenter un fonds qui financera celles qui forment au-delà de cette limite, y compris les entreprises de moins de 10 salariés. Les moyens financiers éventuellement restants seront attribués aux centres de formation interentreprises. L'application de cette mesure connaît un certain nombre d'exceptions. Elle ne concerne ni les branches ayant conclu une convention collective portant sur l'apprentissage, comme la chimie ou le bâtiment, ni les firmes (ou les communes) menacées de faillite, ni les institutions sociales telles que hôpitaux ou écoles. De toute manière, la loi n'entrera pas en vigueur si les représentants du monde économique parviennent à conclure un pacte pour la formation professionnelle substituant un engagement ferme des entreprises à la contrainte légale.

Le projet de loi sur l'impôt apprentissage (Ausbildungsplatzabgabe), annoncé fin 2003, fut fraîchement accueilli. Contestée jusque dans les rangs des sociaux-démocrates, la nouvelle mesure est considérée comme inopérante par $70 \%$ de la population allemande interrogée à ce sujet. Seuls les syndicats soutiennent l'initiative du gouvernement. Le groupe parlementaire chargé de l'ap- 
préciation du projet met en exergue un certain nombre de questions laissées sans réponse :

- quel sera le montant de l'impôt et qui sera chargé de sa perception ? Sera-t-il basé sur les salaires ou le chiffre d'affaires, sachant que le premier cas pénalise les entreprises riches en main-d'œuvre, le second celles riches en capital ?

- quels critères seront appliqués aux régions économiquement faibles?

- comment seront traitées les entreprises qui offrent des places d'apprentissage mais ne trouvent pas de candidats qui conviennent? Ou celles qui ne sont pas autorisées à former des jeunes?

Si les membres du groupe parlementaire, après de longues discussions, ont fini par voter le projet à une faible majorité, les associations patronales ont fait état de leur opposition totale à ce projet. Mises à part les mêmes craintes sur le (dys)fonctionnement du système à mettre en place, elles soulignent surtout les dangers qu'il constitue pour l'apprentissage lui-même. L'instauration de l'impôt risque d'amener les entreprises à ne plus former que pour leurs propres besoins, voire à renoncer à la formation duale. Ce processus pourrait conduire à un déplacement de la formation vers des centres d'apprentissage hors entreprise, considérés comme plus chers, d'une qualité inférieure et conduisant à un plus faible taux d'insertion des jeunes à l'issue de leur formation.

La réponse du gouvernement à ces critiques était de souligner sa volonté de sauvegarder à tout prix les chances des jeunes - de tous les jeunes - de bénéficier d'une formation professionnelle solide, loin des stages d'insertion ou de préprofessionnalisation actuellement offerts aux laissés pour compte. Cet objectif pourrait être atteint soit par l'engagement ferme des entreprises, soit, à défaut de cette garantie, par la contrainte, comme le laissait entendre le chancelier Schröder à maintes reprises.

Bien qu'opposés à une loi qu'ils réprouvent, les représentants patronaux, les $\mathrm{CCl}$ et les chambres de l'artisanat en tête, ne pouvaient se résoudre à s'engager dans la voie d'un engagement volontaire, estimant que l'apprentissage est également l'affaire des écoles, des parents d'élèves et des pouvoirs politiques. Ils se sont néanmoins déclarés prêts à offrir une place d'apprentissage à « tous les candidats volontaires et aptes » à s'engager dans cette voie. Si les deux parties ont finalement réussi à se mettre d'accord sur un provisoire retrait de la loi au profit de l'engagement volontaire, c'est non seulement dû à la réaction négative devant une loi jugée superflue, voire néfaste, mais aussi à cause des craintes du gouvernement. II n'était pas certain, devant l'attitude récalcitrante de certains Länder gouvernés par le SPD, notamment le Schleswig-Holstein, qu'il réussirait à faire passer sa loi devant les instances législatives.

Après s'être affrontés pendant tout le premier semestre 2004, le gouvernement et les fédérations patronales ont signé le 16 juin 2004, à la surprise quasigénérale, un «Pacte national pour la formation et les jeunes professionnels en Allemagne », d'une durée de trois ans. Selon les termes du pacte, les acteurs économiques se fixent l'objectif de rechercher, en moyenne annuelle, 30000 places d'apprentissage de plus afin de compenser au mieux les places d'apprentis supprimées pour des raison économiques et autres, voire d'accroître si possible le nombre de nouvelles places. Les « nouvelles places » en question ne signifient pas « des places supplémentaires». Des places supprimées en raison de faillites ou autres n'entrent pas dans le calcul. Le pacte ne donne pas la garantie d'un nombre donné de places d'apprentissage. En automne, tous les candidats non encore placés seront invités à se soumettre à un test de compétences. Ceux qui ne se déplacent pas - plus de $50 \%$ à en juger par l'expérience du passé - seront rayés des statistiques. A la fin de l'année, les jeunes sans place d'apprentissage recevront une offre de stage d'insertion professionnelle dont les entreprises promettent de créer 25000 de plus par an. Pour ces stages, les entreprises ne se chargeront que des coûts matériels, la rémunération des jeunes étant assurées par une subvention du gouvernement fédéral versée par les ANPE.
Les risques d'une contrainte légale

Réticences des acteurs économiques

Retrait provisoire du projet de loi ... 
Comparé aux objectifs définis dans le projet de loi sur l'impôt apprentissage, le Pacte pour la formation signé entre le gouvernement et les acteurs économiques paraît singulièrement dilué. La notion de mise à disposition garantie d'une place d'apprentissage pour les jeunes a été abandonnée au profit d'une promesse floue de création de postes d'apprentis et de stages qui ne tient compte de l'évolution ni de l'offre ni de la demande en matière de formation duale. La promesse ne se réfère d'ailleurs pas au nombre de nouveaux contrats, mais seulement à la quantité de places proposées par les entreprises. Aucune sanction n'est prévue dans le cadre du pacte en cas de promesse non tenue, à moins qu'on ne considère comme telle la menace de l'application de la loi sur l'impôt apprentissage, comme les Verts se complaisent à le rappeler. Un autre problème crucial est le terme de jeune « volontaire et apte à la formation » introduit par le pacte. II prévoit en effet que tous les jeunes qui n'ont pas trouvé de place d'apprenti à une date butoir donnée doivent passer un contrôle de compétences. S'ils ne se présentent pas à l'examen, ils sont considérés comme "non volontaires », s'ils le ratent, ils sont « inaptes". Dans les deux cas, ils sont ôtés des statistiques nationales. Ces jeunes, dont le nombre peut atteindre les 100000 par an, sont les véritables laissés pour compte du marché de la formation duale. Ils formeront le plus gros des futurs bataillons de chômeurs.

L'ACTUEL HIATUS ENTRE L'OFFRE ET LA DEMANDE dans le domaine de la formation duale est peut-être un phénomène passager. En raison de la situation démographique, le nombre d'élèves quittant les écoles, et donc de candidats, commencera bientôt à refluer. La perspective d'une future pénurie de personnel qualifié rend d'autant plus urgent de pallier, dès maintenant, les dysfonctionnements du marché de la formation duale. Ni l'impôt apprentissage ni le Pacte pour la formation ne semblent de nature à porter remède à la situation des laissés pour compte du système actuel. En attendant une hypothétique embellie de la croissance économique, les acteurs économiques estiment plus judicieux de faire davantage appel aux autres acteurs concernés par la formation duale. Les écoles et les entreprises pourraient coopérer davantage en améliorant les contenus scolaires, en multipliant les stages courts d'initiation à la vie de l'entreprise (dès 14 ou 15 ans) et les contacts entre professeurs et représentants des entreprises pour mieux préparer les élèves à leur entrée dans la vie professionnelle. Les partenaires sociaux, quant à eux, pourraient s'inspirer de l'exemple de la chimie et du bâtiment qui, dans le cadre d'une convention collective, s'entendent librement sur une politique de formation portant non pas sur les contenus (ils sont définis de manière tripartite), mais le nombre de places à créer dans tel ou tel secteur. On peut regretter que ces voies qui font appel à la coopération des acteurs sur le terrain n'aient pas davantage été explorées dans le récent - et assez stérile - débat sur la formation professionnelle. Un débat qui, de surcroît, fait fi de la responsabilité historique des entreprises dans l'effort de formation ; il fondait le succès de la formation duale, jusqu'ici voie royale de l'insertion sociale et professionnelle des jeunes, en garantissant l'adéquation des formations délivrées avec la demande du marché.

\section{Indications bibliographiques}

DeUtSChER BundestAG, « Entwurf eines Gesetzes zur Sicherung und Förderung des Fachkräftenachwuchses und der Berufsausbildungschancen der jungen Generation (Berufsausbildungssicherungsgesetz - BerASichG), BT-Drucksache 15/2820

Dietrich H., KосH S., Stops M., « Ausbildung muss sich lohnen auch für die Betriebe“, IAB Kurzbericht 6/2004

LASSERRE R., LATTARD A., La formation professionnelle en Allemagne. Spécificités et dynamique d'un système, CIRAC, 1993

LESTRADE B., «Emploi-jeunes, une comparaison entre la France et l'Allemagne ? », Allemagne d'aujourd'hui, n'151/2000

Nationaler Pakt für Ausbildung und Fachkräftenachwuchs in Deutschland, 16 juin 2004 (www.bundesregierung.de) 